\title{
MCF-7, ACHN, and A549 Cancer Cells Characterized by Quantitative Magnetic Resonance Imaging In Vitro
}

\author{
Adrian Truszkiewicz $^{1(\mathbb{D})}$, David Aebisher ${ }^{1(\mathbb{D})}$, Dorota Bartusik-Aebisher ${ }^{2, *}(\mathbb{D})$

\begin{abstract}
Department of Photomedicine and Physical Chemistry, Medical College of The University of Rzeszow, Rzeszow, Poland Department of Biochemistry and General Chemistry, Medical College of The University of Rzeszow, Rzeszow, Poland
\end{abstract} \\ Correspondence: author: dbartusik-aebisher@ur.edu.pl (D.B.A.);
}

Scopus Author ID 26029745200

Received: 25.07.2021; Revised: 5.09.2021; Accepted: 10.09.2021; Published: 17.10.2021

\begin{abstract}
To work with cancer cell cultures in vitro at 1.5 Tesla Magnetic Resonance Imaging (MRI), it was necessary to develop dedicated receiver coils. This device allowed to adjust the shape of the tested objects and thus improve the quality of imaging. One of the conditions for this new device was to increase the recorded signal level and reduce the distance between the tested object and the receiving elements of the coil. MCF7 (breast adenocarcinoma, Her-2 positive), ACHN (kidney cancer cells), and A549 (lung cancer cells) were characterized by using magnetic resonance imaging (MRI) in vitro. MRI measurements were performed using the clinical scanner with a 1.5 Tesla magnetic field. MCF-7, ACHN, and A549 cancer cells were characterized by T1 and T2 relaxation times. For MCF-7 cells, the relaxation times T1 and T2 were $2360 \pm 12 \mathrm{~ms}$ and $116 \pm 0.9 \mathrm{~ms}$, respectively. For ACHN cells, the relaxation times T1 and T2 were $1354 \pm 193 \mathrm{~ms}$ and $80 \pm 9 \mathrm{~ms}$, respectively. Values of T1 and T2 for A549 cancer cells cultures were $1527 \pm 59 \mathrm{~ms}$ and $150 \pm 8 \mathrm{~ms}$, respectively. Once an accurate pulse protocol has been established and satisfactory reproducibility was obtained, the determination of relaxation times can be used as a tool to monitor cancer cell cultures using MRI in vitro based on the determination of changes in relaxation times.
\end{abstract}

Keywords: magnetic resonance imaging; relaxation times; cell cultures.

(C) 2021 by the authors. This article is an open-access article distributed under the terms and conditions of the Creative Commons Attribution (CC BY) license (https://creativecommons.org/licenses/by/4.0/).

\section{Introduction}

Over the past few decades, magnetic resonance imaging (MRI) has become one of the most popular broadly defined clinical diagnostics methods. MRI enables the acquisition of morphological, functional, and metabolic parameters in tissue. In addition to a number of diagnostic and clinical applications, MRI can be used for in vitro preclinical, experimental research. MRI - is a phenomenon discovered as one of the last diagnostics methods. Without MRI it is impossible to imagine today's medicine, biology, chemistry, physics, and many other related areas of life. It provides invaluable services in the imaging of pathological changes in both humans and animals. It allows the assessment of internal organs' structure and the metabolic pathways and changes taking place in organs, tissues, and cells. With the constantly evolving software that allows you to extract information important for the doctor from the maze of pixels, more sophisticated techniques and sequences of tests cause MR diagnostics to be constantly accelerating. Multichannel coils contribute to shortening the examination time as well as its improvement. High magnetic fields result in better resolution both in space and in the frequency domain. Scientists and engineers are developing new functionalities in this newly 
discovered technique, against which it will not be possible to say the last word for a long time, and its replacement will require further breakthroughs.

The use of cell cultures and checking their metabolism may allow transferring in vitro studies to in vivo tests on a living organism due to the corresponding cell morphology. Zhang et al. studied various cell lines and the influence of a strong magnetic field on the cell cycle and cell death [1]. The aim of the study is to use MRI to develop a methodology for imaging MCF-7, ACHN, and A549 cancer cell cultures based on changes in $\mathrm{T}_{1}$ and $\mathrm{T}_{2}$ in vitro studies. It is important to improve cellular imaging using MRI, enabling non-invasive imaging of tissues with high sensitivity and time resolution. In MCF-7 breast cancer cells, Her-2 overexpression is associated with a higher rate of tumor cell growth, which is directly related to higher growth factors [2]. The aim of this study was to develop a methodology for measuring ${ }^{1} \mathrm{H}$ MRI of cancer cells in vitro to complete a database allowing for the estimation of qualitative and quantitative changes depending on the type of cells and their condition. These goals were achieved by:

(1) Development of a numerical application that measures relaxation times and its implementation in the MATLAB package (statistics, function approximation, data interpolation, use of DICOM files).

(2) Preparation and performance of ${ }^{1} \mathrm{H}$ MRI measurements with standard substances.

(3) Verification of the calculation algorithm based on the literature and the results of phantom measurements.

(4) Preparation of tumor cell cultures (MCF-7, kidneys, and lungs), which density will enable qualitative and quantitative measurements of ${ }^{1} \mathrm{H}$ MRI to develop appropriate databases.

(5) Development of a method of verification and optimization of results.

In this research, MRI was performed on cell cultures with reproducible results. We used MRI to characterize MCF-7, ACHN, and A549 cancer cell cultures. MRI signal depends on the biochemical properties of the examined cells and physical parameters selected for the examination. Research on cell cultures allows imaging of dynamic and kinetic changes by studying relaxation time $\mathrm{T}_{1}$ and $\mathrm{T}_{2}$. Therefore, in our work, MCF-7, ACHN, and A549 cancer cells were characterized using relaxation time.

\section{Materials and Methods}

\subsection{Cell cultures.}

The MCF-7 cell line (American Type Culture Collection, VA, USA) was purchased from Sigma Aldrich (MO, USA). For culturing this cell line, EMEM medium (EBSS) $+2 \mathrm{mM}$ glutamine $+1 \%$ NEAA indispensable amino acids $+10 \%$ FBS fetal bovine serum were used. In addition, we used the A549 lung cancer cell line (American Type Culture Collection, VA, USA) and the ACHN kidney cancer cell line (American Type Culture Collection, VA, USA). Both cell lines were grown under standard conditions: $37^{\circ} \mathrm{C}, 5 \% \mathrm{CO}_{2}$, and $95 \%$ humidity. The culture medium consisted of modified Eagle Dulbecco's medium (Sigma-Aldrich, MO, USA), modified Dulbecco Eagle Eagle's nutrient mix F-12 Ham medium (Sigma-Aldrich, MO, USA), fetal bovine serum (Biochrom, Germany), and penicillin-streptomycin (Sigma-Aldrich, MO, USA). Cells were counted using Muse Cell Analyzer (Merck Millipore, MA, USA). 


\subsection{MRI quantitative.}

The experiments were performed using Optima MR360 magnetic resonance from General Electric Healthcare (Milwaukee, Wisconsin, USA) with 1.5 Tesla field strength, software version SV23. The cell cultures in the vials were placed in the center of the magnetic resonance magnet. Prepared samples were scanned using the Fast Spin-Echo (FSE) sequence with axial projection using a small flex coil. The following scanning parameters were used: FOV field of view $=10 \times 10 \mathrm{~cm}$; Matrix $=320 \times 224 ;$ NEX $=2.0$; Slice Thickness $=1.0 \mathrm{~mm}$; Spacing $=0.5 \mathrm{~mm}$.

T1 relaxation time measurements were made in 13 steps with a repetition time (TR) in the range of $50 \div 15000 \mathrm{~ms}(31,60,100,200,500,700,1000,1500,2000,3000,5000,10000$, $15000 \mathrm{~ms}$ ) with a constant echo time (TE) of $3 \mathrm{~ms}$. However, in the case of T2 relaxation time, TE time varied in the range of $10 \div 250 \mathrm{~ms}(10,20,30,42,68,85,102,130,150,200,250$, $300,447.4 \mathrm{~ms}$ ). TE time was unchanging and amounted to $10,000 \mathrm{~ms}$. The figure 1 shows examples of DICOM images from the determination of relaxation times T1 and T2. The left part includes images made with the following TR scanning parameters: 32, 700, 2000, 5000, $15000 \mathrm{~ms}$, respectively, with a constant echo time $\mathrm{TE}=3 \mathrm{~ms}$. The right part shows images made with the following TE parameters: 10, 68, 130, 250, $447.4 \mathrm{~ms}$, while maintaining a constant repetition time $\mathrm{TR}=10,000 \mathrm{~ms}$.

\section{Results and Discussion}

The study used the OPTIMA 360MR magnetic resonance system manufactured by General Electric Healthcare. It is a device based on a 1.5 Tesla superconducting magnet. The parameters of the gradient system, which are the amplitude and the slope speed, are respectively $33 \mathrm{mT} / \mathrm{m}$ and $120 \mathrm{~T} / \mathrm{m} / \mathrm{s}$. It is equipped with a set of coils to diagnose the entire human body and diagnostic stations, allowing for the analysis of the obtained images for medical diagnosis. A specialized software package with a system for researching the field of elastography makes it a very good system for imaging diagnostics of the human body.

Analyzing scientific articles about cell culture research, it can be concluded that they are the domain of high-field systems [3,4]. However, it should be said that at present, the exact influence of the magnetic field on the human body is not known yet, and even more so on small and very sensitive structures such as single cells. Rapid changes in the gradients in the system cause a change in the magnetic field and thus the flow of small amounts of current inside the examined structures. At present, there is insufficient information on this effect on the tested cells. It may turn out that the distant effects of the current or the magnetic field itself may lead to erroneous conclusions.

Figure 1 presents 5 of 12 selected scans in acquired sequences for determining $\mathrm{T}_{1}$ time. The image located on the right side is selected scans necessary to determine the $T_{2}$ time. The images shown on the left and right are the same tubes scanned in the same planes to compare the images.

\subsection{MCF-7 lung cancer.}

The presented Figure 1 shows the distribution of T1 and T2 times in the tested samples containing MCF-7 breast cancer cells. The image clearly shows the area with the shorter T1 time, which is the area occupied by cells. Measurement was made in the middle of the area representing the cells. It should be noted that the image contains artifacts - they appear in the 
wall area of the test tubes as well as on the fluid-air interface. Above is the distribution of $\mathrm{T}_{1}$ times in samples containing MCF-7 breast cancer cell cultures.

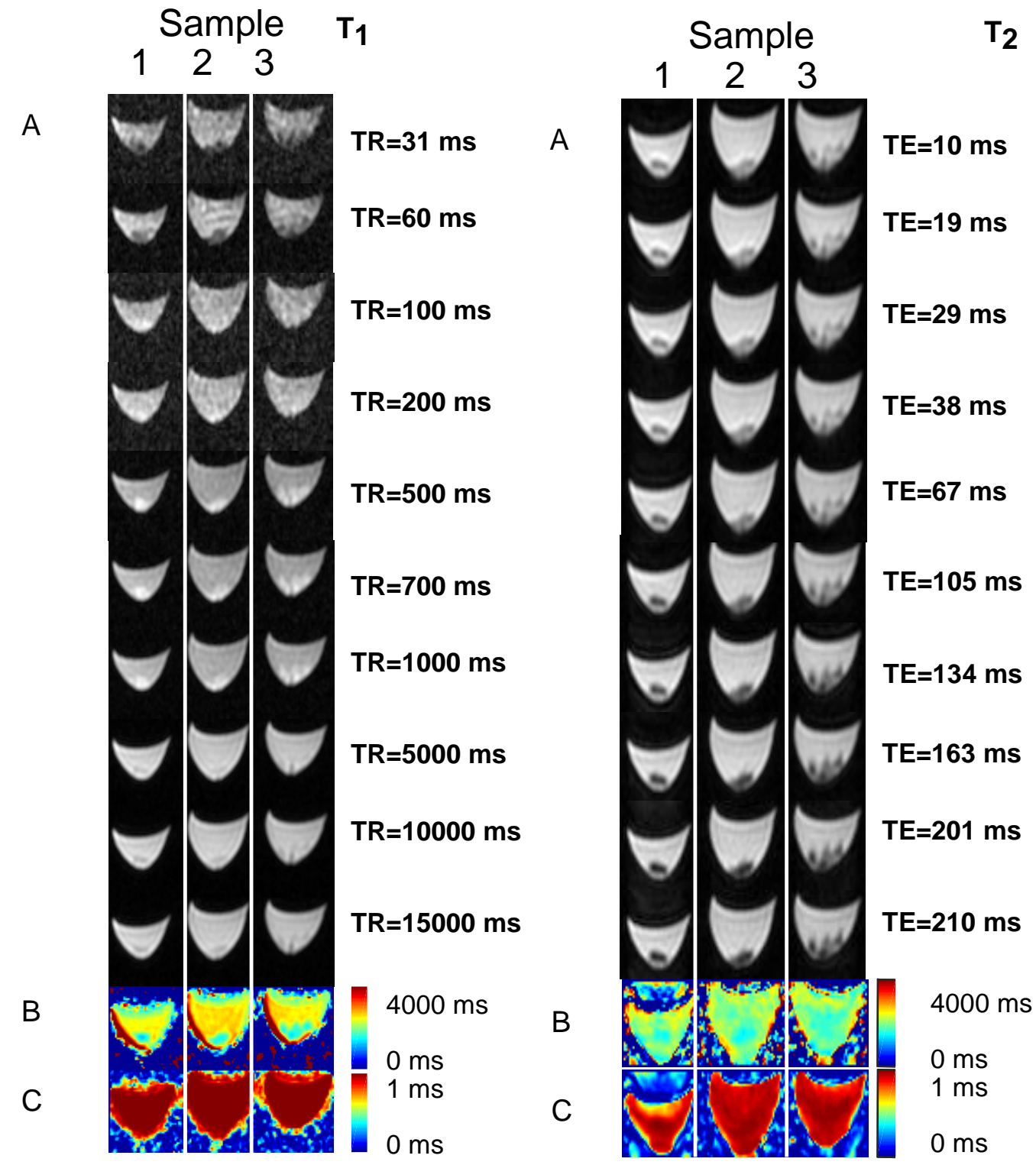

Figure 1. The methodology of $T_{1}$ and $T_{2}$ measurements of MCF-7 cells.

Table 1. $\mathrm{T}_{1}$ and $\mathrm{T}_{2}$ of MCF-7 cell cultures.

MCF-7 cell cultures (Figure 1)

\begin{tabular}{c|c|c|c}
\hline & Sample A & Sample B & Sample C \\
\hline $\mathbf{T}_{\mathbf{1}} \mathbf{m s}$ & $2773,64 \pm 22,19$ & $2237,07 \pm 17,9$ & $2068,05 \pm 16,54$ \\
\hline $\mathbf{T}_{\mathbf{2}} \mathbf{m s}$ & $115,30 \pm 0,92$ & $126,32 \pm 1,01$ & $105,49 \pm 0,84$
\end{tabular}

You can clearly see the border of the area where the cells are located. The range of $\mathrm{T}_{1}$ times presented is in the range of 1 to 4000 . This range completely covers the range of changes in longitudinal relaxation times in cell cultures. It can be unequivocally stated that the fluid constituting the culture medium has a significantly longer $\mathrm{T}_{1}$ time compared to the cell area. This figure shows the red areas on the right of the tubes - these are artifacts. The calculated time $\mathrm{T}_{1}$ takes values up to $7000 \mathrm{~ms}$. Similarly, the color change in the upper part of the fluid at the air border should be considered artifacts. They manifest themselves with increased noise. A solid blue background around colored images needs some explanation. These are places where the R2 factor does not exceed 0.4 , which is a sign of a very poor fit. To remove noise from the image, the $T_{1}$ values specified with $\mathrm{R} 2$ below 0.4 have been replaced by a value of 0 
ms. Distribution of the fit factor of the function approximating T1 times to the measured data. Graphical presentation of this parameter allows the reader to assess the accuracy of the individual areas of the image. It is visible that the places in the studied space, the characteristics of which correspond to the characteristics of the longitudinal relaxation phenomenon, are defined with a coefficient close to one. The coefficient of determination measures the degree to which the approximating function, i.e., the mathematical model, fits the sample, i.e., the signal intensity value in individual scans. The discerning reader will probably consider the low resolution of the images. It results from the magnification of the image. The actual dimensions of the test areas presented in the image sections are approx. $30 \mathrm{~mm} \times 10 \mathrm{~mm}$, which corresponds to approx. 140 x 40 pixels. This value shows how accurately the longitudinal relaxation time was estimated based on measurement data. The high $\mathrm{R} 2$ coefficient within the tubes confirms that the curve that approximates the measurement data has been correctly determined.

\subsection{A549 lung cancer.}

The studies were carried out on A549 lung cancer. The measurements needed to determine longitudinal and transverse relaxation were made. The next stage was the data analysis, which carried out a thorough analysis of the results obtained, to determine the relaxation times $T_{1}$ and $T_{2}$ in the tested samples, which allowed the characteristics of the tested sample. The analysis of the received data was performed using a licensed MATLAB package.

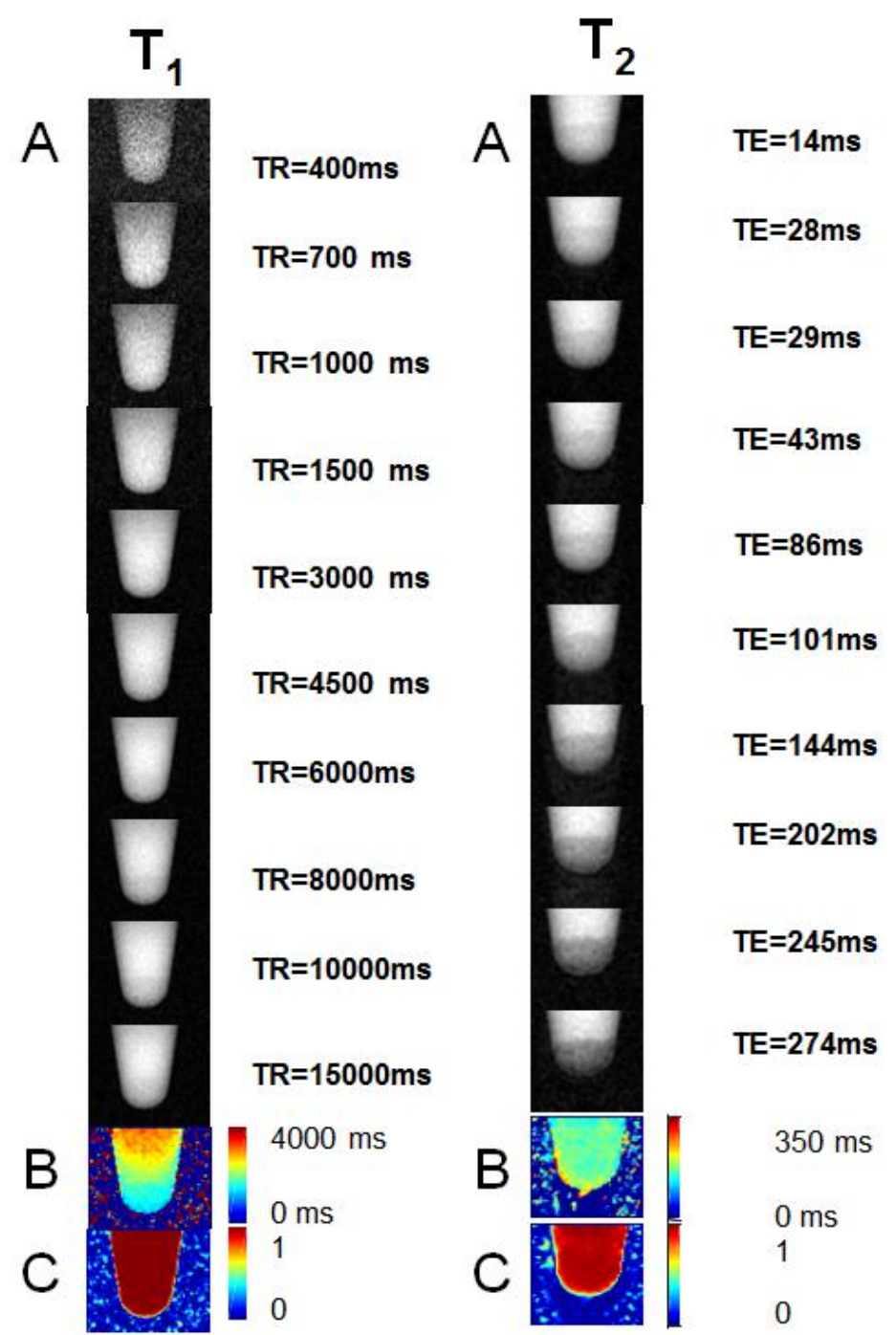

Figure 2. $T_{1}$ relaxation time of A549 cancer cell cultures. 
Figure 2 presents the results that were obtained as a result of calculating the relaxation times $\mathrm{T}_{1}$ and the factor fitting $\mathrm{R}^{2}$ to the culture of the A549 lung cell line. Similar to Figure $2 \mathrm{~B}$, the picture shows $T_{1}$ time maps. Also, in this case, the artifact related to the tube boundary is visible, which is marked in red, symbolizing the upper limit of the assumed timescale $T_{1}$. In this case, it was also limited only to the imaging of pixels for which the fit factor was in the range ( 0.4 to 1$)$. In this case, the study was performed in the coronal plane. Table 2 shows the results of relaxation times that were obtained for the MCF-7 breast cancer cell line. Table 2 shows the results of relaxation times for A549 lung cancer.

Table. 2. $\mathrm{T}_{1}$ and $\mathrm{T}_{2}$ relaxation times for A549 lung cancer cell line.

\begin{tabular}{c|c} 
& Samples $(\mathrm{n}=6)$ \\
\hline $\mathbf{T}_{\mathbf{1}}[\mathrm{ms}]$ & $1527 \pm 59$ \\
\hline $\mathbf{T}_{\mathbf{2}}[\mathrm{ms}]$ & $150 \pm 8$
\end{tabular}

\subsection{ACHN kidney cancer.}

The following table compares $\mathrm{T}_{1}$ and $\mathrm{T}_{2}$ relaxation time values for samples with A549 lung cancer cells and ACHN kidney cancer.

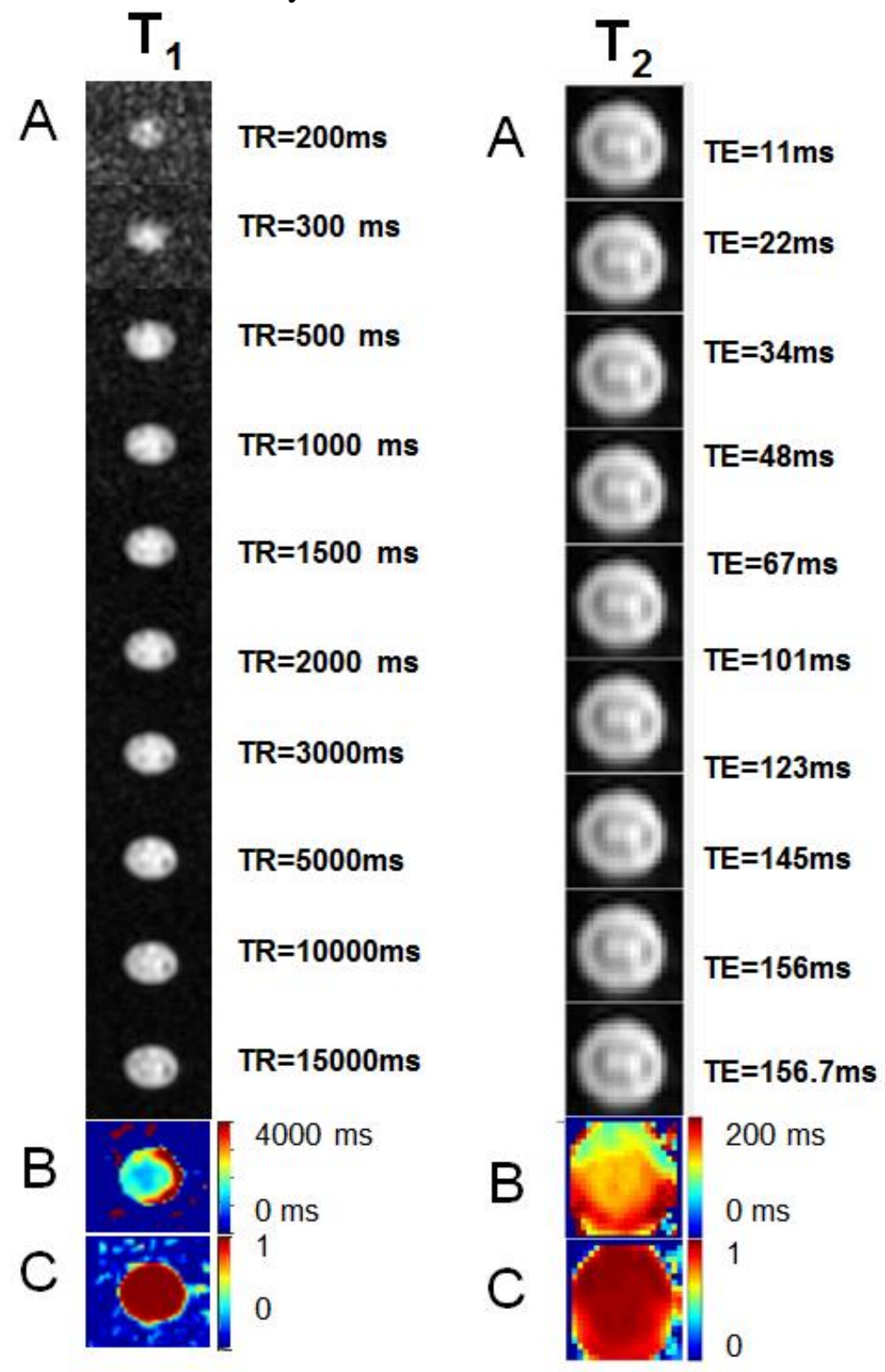

Figure 3. $T_{1}$ relaxation time of ACHN cancer cell cultures. 
Table. 3. $T_{1}$ and $T_{2}$ relaxation times for $A C H N$ lung cancer cell line.

\begin{tabular}{c|c} 
& Samples $(\mathrm{n}=6)$ \\
\hline $\mathbf{T}_{\mathbf{1}}[\mathbf{m s}]$ & $1354 \pm 193$ \\
\hline $\mathbf{T}_{\mathbf{2}}[\mathbf{m s}]$ & $80 \pm 9$
\end{tabular}

It should be emphasized that the factory coils, much more complicated in their construction, perfectly illustrate the human body; however, when it is necessary to record signals from small objects, they are not always optimal. Often, dedicated surface coils allow for imaging with better parameters - their disadvantage is usually a small area that can be examined. The highly non-linear characteristic as a function of the distance from the coil means that only a small range of the area is possible for imaging. There are many publications in the literature on the design of experimental coils. Most often, surface coils are used to image nearsurface areas and small objects. Readers who want to see examples of this type of receiving system's solutions can be offered positions in which researchers used these types of electronic systems for magnetic resonance microscopy (MRM) [5-8]. It should be added that on the publishing market, you can also find books about MR coils [9]. And although high-field MR systems are the domain of experimental imaging, and in particular of small objects or structures not of a typical shape, 1.5 Tesla devices can also be used in research. This work proves this because it has been made entirely on the basis of such a medium-field MR system.

Several different receiver systems were developed during the implementation of the tasks, which served as coils for recording signals from the tested structures. The tests were performed with both surface and solenoid coils. Since they had a perfect homogeneity of the field, the latter allowed them to obtain very good images. However, covering the assumed test space with such a structure poses many difficulties as far as the electronic structure is concerned. Extending the coil causes an increase in inductance and thus a change in impedance parameters for the resonance frequency. The necessity to ensure an appropriate wave impedance requires the use of impedance matching circuits. This, in turn, leads to a loss of signal.

The basic assumption of the first coil was the possibility of testing test tubes with a maximum diameter of approx. 20 and a length of approx. $50 \mathrm{~mm}$. Its structure is based on a copper sheet section $0.2 \mathrm{~mm}$ thick and $5 \mathrm{~mm}$ wide. The choice of material was determined by the need to minimize losses. At the frequency of $63.885 \mathrm{MHz}$, there is a skin effect which causes that the current flows in the conductor only through the outer part of the wire. For copper under these conditions, the skin depth is only $8.34 \mu \mathrm{m}$. This means that in practice, only this part of the electric current flows. It should be added that the function describing the current density distribution as a function of the radius of the conductor is very steep, but it is continuous. This epidermal depth is the point on the radius of the circular conductor where the current drops to approx. $37 \%$ of the maximum value:

$$
J_{\S}=J_{\max } \cdot e^{-\mathbb{1}}
$$

This problem is very well described in world literature and goes beyond the scope of this work [10-17].

The construction uses capacitors specially made for applications in MR systems. The entire system was tuned to the central frequency with the RIGOL DSA815 TG Spectrum Analyzer. The mechanical structure of the coil is based on Teflon. This synthetic fluoropolymer is perfect for making coil housing where stiffness, high resistance, and low leakage are required. 
A phantom containing water and plastic capillaries was prepared to evaluate the operation of the coil. The dimensions of the elements included in the phantom (Figure 4) are the outer diameter of the glass tube $16.5 \mathrm{~mm} \pm 0.05 \mathrm{~mm}$, the inner diameter of the glass tube is $13.5 \mathrm{~mm} \pm 0.05 \mathrm{~mm}$, the outer diameter of the capillary $2 \mathrm{~mm} \pm 0.05 \mathrm{~mm}$, the thickness of the capillary wall $0.3 \mathrm{~mm} \pm 0.05 \mathrm{~mm}$, inner diameter of the capillary $1.4 \mathrm{~mm} \pm 0.05 \mathrm{~mm}$. The glass tube was half full with capillaries.

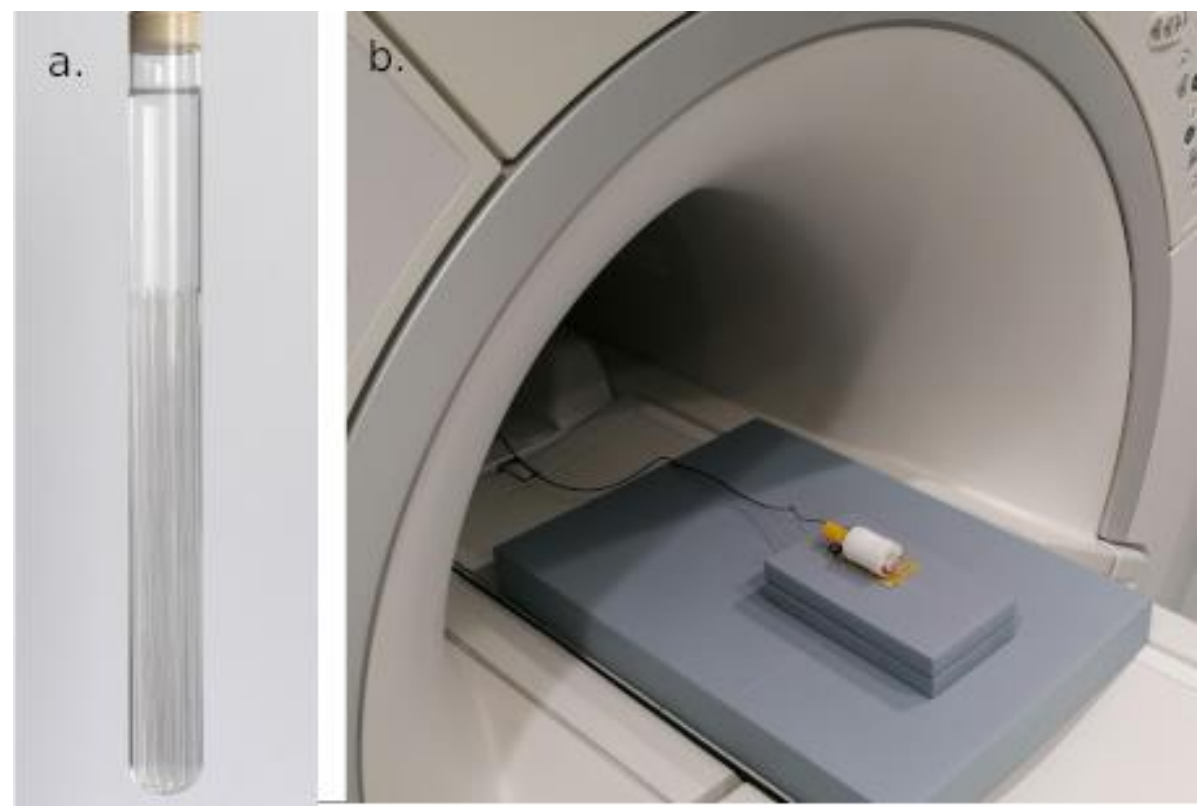

Figure 4. (a) Phantom for testing coils; (b) appearance of the coil mounted to the MRI system, prepared for phantom imaging (house-made).

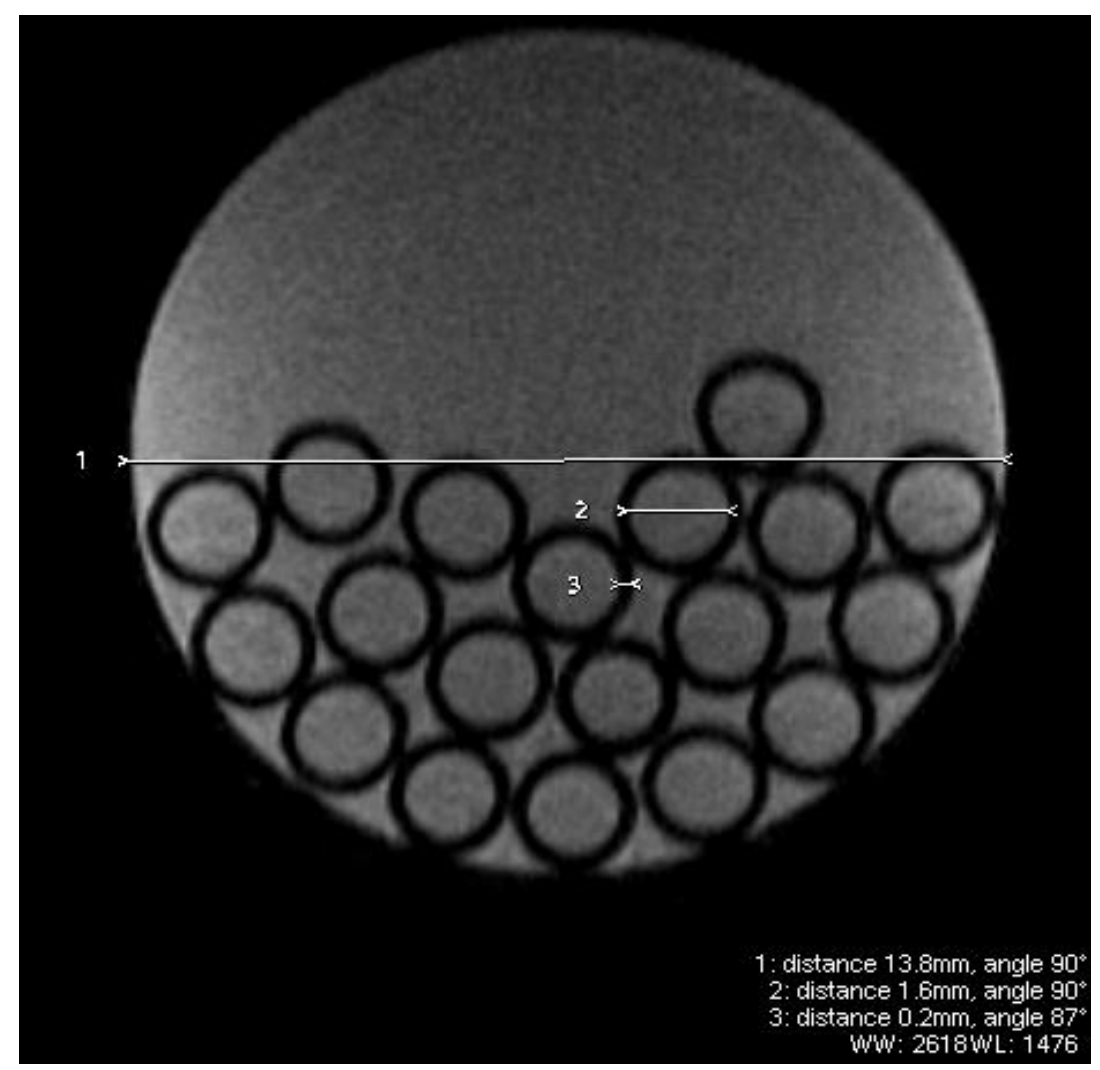

Figure 5. The result of scanning the phantom with an experimental coil with the marked dimensions obtained at a medical diagnostic station (own elaboration). 
As shown in Figure 4, the coil allowed imaging the phantom with a modified wrist examination protocol. The minimum FOV was $4 \mathrm{~cm}$, and the acquisition resolution was $512 \times 152$. Such imaging conditions allow obtaining spatial resolution on the level of about $78 \mathrm{um}$. Such a high resolution obtained in this system also has some disadvantages, namely reducing the pixel size is associated with increasing the share of noise in the resulting image. Nevertheless, the attempt to increase the spatial resolution in the study of this group of objects is fully justified.

Figure 5 shows the results of the phantom scan and the obtainable MR images. The very good quality of the obtained image deserves attention. This coil also makes it possible to obtain spectroscopic images.

The assumptions for the work were to develop an application that would allow the calculation of longitudinal and transverse relaxation times based on the data obtained as a result of MR signal acquisition. An integral part of the work was an application developed and written in MATLAB. The operation of the application was based on the GUI user interface in which DICOM files are downloaded, processed, and in the final stage, the results are displayed. The program allows you to work in four modes:

1. Measurement of $\mathrm{T} 1$ and $\mathrm{T} 2$ time based on an average value of the region of interest. The result is the numerical value of Times $\mathrm{T} 1$ and $\mathrm{T} 2$ and the value of the adjustment coefficient R2;

2. Measurement of T1 and T2 time based on the values of individual pixels in the profile selected and marked on the DICOM image. In the model, the result consists of three charts presenting:

- T1 or T2 time chart in the selected profile;

- graph of the R2 fit factor;

- signal intensity graph in the analyzed profile;

3. T1 and T2 time measurement for the selected area. This mode provides a pixel-topixel map of the T1 as well as T2 times. In addition, it is also possible to obtain images of the R2 coefficient;

4. Measurement of T1 and T2 time based on the mean value of the region of interest with the difference that all raw data and results are saved to an ASCII text file. This functionality allows for easy documentation as well as post-processing.

Based on the performed tests, it can be stated that it turns out to be important to select the necessary non-invasive method for the quantitative and qualitative assessment of research in imaging cell cultures. In this study, a coil to study small objects, which are undoubtedly cell cultures, was used. The small flex surface coil can be used for testing small objects because they are characterized by high resolution and sensitivity, which improves the received signal in vitro tests. It should be noted that the coil has a heterogeneous signal intensity, which should be taken into account when testing cultures. In cell culture studies, attention should be paid to the resolution and sensitivity of the coil, which significantly decreases with the distance of the tested object from the coil. In the first study in 1977, Wagh et al. presented measurements of longitudinal relaxation time in cell cultures [18]. Since then, there have been many reports on the use of the MRI method for experimental research. In scientific studies, Haedicke et al. we can read about the $T_{1}$ contrast agent based on manganese porphyrin, MnEtP used for labeling and tracking stem cells using MRI 3T [19]. Jasmin also in vitro studies described the use of SPION for labeling mesenchymal stem cells and assessing their efficacy and cytotoxicity using the MRI method [20]. In contrast, Pothirajan et al., in their work, evaluated the possibility of 
using MRI to monitor cartilage growth by non-invasive assessment of cells and extracellular matrix (ECM) using relaxation times $\mathrm{T}_{1}$ and $\mathrm{T}_{2}$ [21]. Mohhimi et al. assessed gadolinium oxide cytotoxicity by implementing polymers and nanoparticles in Hepa 1-6 cell lines by assessing changes in $\mathrm{T}_{1}$ longitudinal relaxation [22]. $\mathrm{T}_{1}$ relaxation time in the human lung based on a review of Dietrich et al. for 1.5 Tesla resonance, it is about $1200 \mathrm{~ms} \pm 150 \mathrm{~ms}$, while breathing pure oxygen is about $1050 \mathrm{~ms}$ (Dietrich et al. 2017). Alamidi et al., based on the conducted research, presented the T1 relaxation time in smokers' lungs, which decreased with age [23]. There are also studies talking about the values of $\mathrm{T} 1$ and $\mathrm{T} 2$ relaxation times for pathological tissues [24]. Sobhani et al. used manganese zinc ferrite in their research as a contrast agent to detect mouse breast cancer cells [25]. Beall et al. published results showing differences in $\mathrm{T}_{1}$ and $\mathrm{T}_{2}$ relaxation times for healthy and cancerous epithelial breast cells in mouse cultures, which were $916 \pm 24 \mathrm{~ms}$ and $1155 \pm 42 \mathrm{~ms}$, respectively [26]. Khaniabadi et al. used superparamagnetic iron oxide nanoparticles (SPION) and using the spin-echo sequence, obtained T2-dependent images based on which breast cancer cells (MCF-7) were detected [27]. The use of coated nanoparticles (NP), for example, SPION coated with trastuzumab and indocyanine green (ICG) can be successfully used as drug carriers for HER2 overexpressing breast cancer as demonstrated in in vitro and in vivo studies by Luo et al. [28]. Similar studies were performed by Khaniabadi et al., Who showed in vitro studies that superparamagnetic iron oxide nanoparticles conjugated to the C595 monoclonal antibody (SPION-C595) reduce T2 relaxation time $76 \%$, which may prove to be an effective method for detecting breast cancer cells MCF7 [29]. Gunanathan et al. in their research they proposed contrast agents containing pyridine containing Gd (III) and Eu (III) (EPTA-Gd / Eu), water-soluble, for magnetic resonance imaging, which effectively reduced $T_{1}$ and $T_{2}$ relaxation times at the location of the estrogen receptor [30].

There are several scientific reports describing changes in relaxation time in the kidneys; for example, McLachlan and Hamilton using the $\mathrm{T}_{1}$ relaxation time examined the effect of Sarcoma-180 cancer on blood and tissues in mouse studies, based on the research found an increase in relaxation times of kidney and blood [31]. Escanye et al. investigated the relationship between longitudinal relaxation in healthy and tumor tissues of mice and Larmor frequencies, but no consistent results were obtained [32]. Ross et al. using ${ }^{31} \mathrm{P}$ NMR they implemented studies on renal biochemistry, based on which it was found that $\mathrm{T}_{1}$ relaxation time is different for the renal cortex and spinal cord, which may be due to different lipid composition [33]. Takeda et al. confirmed that renal relaxation times in vivo studies could be calculated based on low magnetic field MRI results [34]. An et al. used $\mathrm{T}_{2}$ relaxation time to assess ability (FA-MAN) in assessing glioma imaging in vivo [35].

\section{Conclusions}

The study used the OPTIMA 360MR magnetic resonance system manufactured by General Electric Healthcare. It is a device based on a $1.5 \mathrm{~T}$ superconducting magnet. The parameters of the gradient system, which are the amplitude and the slope speed, are respectively $33 \mathrm{mT} / \mathrm{m}$ and $120 \mathrm{~T} / \mathrm{m} / \mathrm{s}$. It is equipped with a set of coils to diagnose the entire human body and diagnostic stations, allowing for the analysis of the obtained images for medical diagnosis. A specialized software package with a system for researching the field of elastography makes it a very good system for imaging diagnostics of the human body.

Analyzing scientific articles about cell culture research, it can be concluded that they are the domain of high-bay systems. However, it should be said that the exact influence of the 
magnetic field on the human body is not known yet, and even more so on small and very sensitive structures such as single cells. Rapid changes in the gradients in the system cause a change in the magnetic field and thus the flow of small amounts of current inside the examined structures. At present, there is insufficient information on this effect on the tested cells. It may turn out that the distant effects of the current or the magnetic field itself may lead to erroneous conclusions. For several years, the number of works on the impact of work, especially with high-pressure systems on the body of employees or patients themselves, has been increasing

\section{Funding}

This research was funded by the National Center of Science NCN Grant OPUS-13 number 2017/25/B/ST4/02481.

\section{Acknowledgments}

Dorota Bartusik-Aebisher acknowledges support from the National Center of Science NCN (New drug delivery systems-MRI study, Grant OPUS-13 number 2017/25/B/ST4/02481).

\section{Conflicts of Interest}

The authors declare no conflict of interest.

\section{References}

1. Zhang, L.; Ji, X.; Yang, X.; Zhang, X. Cell type- and density-dependent effect of $1 \mathrm{~T}$ static magnetic field on cell proliferation. Oncotarget 2017, 8, 13126-13141, https://doi.org/10.18632/oncotarget.14480.

2. Bakhsheshian, J.; Strickland, B.A.; Mack, W.J.; Zlokovic, B.V. Investigating the blood-spinal cord barrier in preclinical models: a systematic review of in vivo imaging techniques. Spinal Cord. 2021, 59, 596-612, https://doi.org/10.1038/s41393-021-00623-7.

3. Bartusik, D.; Tomanek, B.; Lattová, E.; Perreault, H.; Fallone, G. Combined treatment of human MCF-7 breast carcinoma with antibody, cationic lipid and hyaluronic acid using ex vivo assays. J. Pharm. Biomed. Anal. 2010, 51, 192-201, https://doi.org/10.1016/j.jpba.2009.07.032.

4. Bartusik, D.; Tomanek, B. Detection of fluorine labeled herceptin using cellular ${ }^{19} \mathrm{~F}$ MRI ex vivo. J. Pharm. Biomed. Anal. 2010, 51, 894-900, https://doi.org/10.1016/j.jpba.2009.10.008.

5. Hermann, P.; Appleby, B.; Brandel, J.P.; Caughey, B.; Collins, S.; Geschwind, M.D.; Green, A.; Haïk, S.; Kovacs, G.G.; Ladogana, A.; Llorens, F.; Mead, S.; Nishida, N.; Pal, S.; Parchi, P.; Pocchiari, M.; Satoh, K.; Zanusso, G.; Zerr, I. Biomarkers and diagnostic guidelines for sporadic Creutzfeldt-Jakob disease. Lancet Neurol. 2021, 20, 235-246, https://doi.org/10.1016/S1474-4422(20)30477-4.

6. Seo, Y. High spatial resolution magnetic resonance imaging of insects covered with a hard exoskeleton. Concepts Magn Reson Part B. 2018; 48B, e21366, https://doi.org/10.1002/cmr.b.21366.

7. Kwok, W.E.; You, Z.; Monu. J.; Seo, G.; Ritchlin C. High-resolution uniform MR imaging of finger joints using a dedicated RF coil at 3T. J. Magn. Reson. Imaging. 2010, 31, 240-247, https://doi.org/10.1002/jmri.21984.

8. Jasiński, K.; Młynarczyk, A.; Latta, P.; Volotovskyy, V.; Węglarz, W.P.; Tomanek, B. A volume microstrip RF coil for MRI microscopy. Magn. Reson. Imaging 2012, 30, 70-77, https://doi.org/10.1016/j.mri.2011.07.010.

9. Vaughan, J.T.; Griffiths, J.R. RF Coils for MRI 2012, John Wiley \& Sons Ltd.

10. Abu-Rumeileh, S.; Capellari, S.; Stanzani-Maserati, M.; Polischi, B.; Martinelli, P.; Caroppo, P.; Ladogana, A.; Parch,i P. The CSF neurofilament light signature in rapidly progressive neurodegenerative dementias. Alzheimers Res Ther. 2018, 10, 3, https://doi.org/10.1186/s13195-017-0331-1.

11. Andelovic, K.; Winter, P.; Jakob, P.M.; Bauer, W.R.; Herold, V.; Zernecke, A. Evaluation of Plaque Characteristics and Inflammation Using Magnetic Resonance Imaging. Biomedicines 2021, 9, 185, https://doi.org/10.3390/biomedicines9020185. 
12. Ferrigno, R.; Hasenmajer, V.; Caiulo, S.; Minnetti, M.; Mazzotta, P.; Storr, H.L.; Isidori, A.M.; Grossman, A.B.; De Martino, M.C.; Savage, M.O. Paediatric Cushing's disease: Epidemiology, pathogenesis, clinical management and outcome. Rev Endocr Metab Disord. 2021, https://doi.org/10.1007/s11154-021-09626-4.

13. Chin, F.; Chou, R.; Waqas, M,; Vakharia, K.; Rai, H.; Levy, E.; Holmes, D. Efficacy of prayer in inducing immediate physiological changes: a systematic analysis of objective experiments. J Complement Integr Med. 2021, https://doi.org/10.1515/jcim-2020-0075.

14. van der Krogt, J.M.A.; van Binsbergen, W.H.; van der Laken, C.J.; Tas, S.W. Novel positron emission tomography tracers for imaging of rheumatoid arthritis. Autoimmun Rev. 2021, 20, 102764, https://doi.org/10.1016/j.autrev.2021.102764.

15. Bartusik-Aebisher D.; Ożóg Ł.; Aebisher D. Alternative methods of photodynamic therapy and oxygen consumption measurements-A review. Biomed Pharmacother. 2021, 134, 111095 , https://doi.org/10.1016/j.biopha.2020.111095.

16. Martins, D.; Rademacher, L.; Gabay, A.S.; Taylor, R.; Richey, J.A.; Smith, D.V.; Goerlich, K.S.; Nawijn, L.; Cremers, H.R.; Wilson, R.; Bhattacharyya S.; Paloyelis Y. Mapping social reward and punishment processing in the human brain: A voxel-based meta-analysis of neuroimaging findings using the social incentive delay task. Neurosci Biobehav Rev. 2021, 122, 1-17, https://doi.org/10.1016/j.neubiorev.2020.12.034.

17. Meca-Lallana, V.; Berenguer-Ruiz, L.; Carreres-Polo, J.; Eichau-Madueño, S.; Ferrer-Lozano, J.; Forero, L.; Higueras, Y.; Téllez Lara, N.; Vidal-Jordana, A.; Pérez-Miralles, F.C. Deciphering Multiple Sclerosis Progression. Front Neurol. 2021, 12, 608491, https://doi.org/10.3389/fneur.2021.608491.

18. Wagh, U.V.; Kasturi, S.R.; Chaughule, R.S.; Shah, S.S.; Ranade, S.S. Studies on proton spin-lattice relaxation time (T1) in experimental cell cultures. Physiol. Chem. Phys. 1977, 9, 167-174.

19. Haedicke, I.E.; Loai, S.; Cheng, H.M. An Efficient $T_{1}$ Contrast Agent for Labeling and Tracking Human Embryonic Stem Cells on MRI. Contrast Media Mol Imaging 2019, 3475786, https://doi.org//10.1155/2019/3475786.

20. Jasmin. In vitro Labeling Mesenchymal Stem Cells with Superparamagnetic Iron Oxide Nanoparticles: Efficacy and Cytotoxicity. Methods Mol. Biol. 2020, 2118, 235-250, https://doi.org/10.1007/978-1-07160319-2_18.

21. Pothirajan, P.; Dorcemus, D.; Nukavarapu, S.; Kotecha, M. True MRI assessment of stem cell chondrogenesis in a tissue engineered matrix. Annu Int Conf IEEE Eng Med Biol Soc 2014, 3933-3936, https://doi.org/10.1109/EMBC.2014.6944484.

22. Moghimi, H.; Zohdiaghdam, R.; Riahialam, N.; Behrouzkia, Z. The Assessment of Toxicity Characteristics of Cellular Uptake of Paramagnetic Nanoparticles as a New Magnetic Resonance Imaging Contrast Agent. Iran. J. Pharm. Res. 2019, 18, 2083-2092, https://doi.org/10.22037/ijpr.2019.1100823.

23. Alamidi, D.F.; Kindvall, S.S.; Hubbard Cristinacce, P.L.; McGrath, D.M.; Young, S.S.; Naish, J.H.; Waterton, J.C.; Wollmer, P.; Diaz, S.; Olsson, M.; Hockings, P.D.; Lagerstrand, K.M.; Parker, G.J.; Olsson, L.E. $\mathrm{T}_{1}$ Relaxation Time in Lungs of Asymptomatic Smokers. PLoS One 2016, 11, e0149760, https://doi.org/10.1371/journal.pone.0149760.

24. Akber, S.F. Water proton relaxation times of pathological tissues. Physiol Chem Phys Med NMR. 2008, 40, $1-42$.

25. Sobhani, T.; Shahbazi-Gahrouei, D.; Rostami, M.; Zahraei, M.; Farzadniya, A. Assessment of ManganeseZinc Ferrite Nanoparticles as a Novel Magnetic Resonance Imaging Contrast Agent for the Detection of 4T1 Breast Cancer Cells. J Med Signals Sens. 2019, 9, 245-251, https://dx.doi.org/10.4103\%2Fjmss.JMSS_59_18.

26. Beall, P.T.; Asch, B.B.; Chang, D.C.; Medina, D.; Hazlewood, C.F. Distinction of normal, preneoplastic, and neoplastic mouse mammary primary cell cultures by water nuclear magnetic resonance relaxation times. $J$ Natl Cancer Inst. 1980, 64, 335-8, https://doi.org/10.1093/jnci/64.2.335.

27. Khaniabadi, M.P.; Shahbazi-Gahrouei, D.; Jaafar, M.S.; Majid, A.M.S.A.; Moradi Khaniabadi, B.; ShahbaziGahrouei, S. Magnetic Iron Oxide Nanoparticles as T2 MR Imaging Contrast Agent for Detection of Breast Cancer (MCF-7) Cell. Avicenna J Med Biotechnol. 2017, 9, 181-188.

28. Luo, X.J.; Li, J.; Zhou, C.W. Preclinical application of MR and fluorescent dual-modality imaging combined with photothermal therapy in HER-2 positive breast cancer. Zhonghua Zhong Liu Za Zhi. 2018, 40, 587-593, https://doi.org/10.3760/cma.j.issn.0253-3766.2018.08.005.

29. Khaniabadi, M.P.; Shahbazi-Gahrouei, D.; Malik Shah Abdul Majid, A.; Suhaimi Jaafar, M.; Moradi Khaniabadi, B.; Shahbazi-Gahrouei, S. In vitro Study of SPIONs-C595 as Molecular Imaging Probe for 
Specific Breast Cancer (MCF-7) Cells Detection. Iran Biomed J. 2017, 21, 360-8, https://doi.org/10.18869/acadpub.ibj.21.6.360.

30. Gunanathan, C.; Pais, A.; Furman-Haran, E.; Seger, D.; Eyal, E.; Mukhopadhyay, S.; Ben-David, Y.; Leitus, G.; Cohen, H.; Vilan, A.; Degani, H.; Milstein, D. Water-soluble contrast agents targeted at the estrogen receptor for molecular magnetic resonance imaging. Bioconjug. Chem. 2007, 18, 1361-5, https://doi.org/10.1021/bc700230m.

31. Nisar, S.; Bhat, A.A.; Hashem, S.; Yadav, S.K.; Rizwan, A.; Singh, M.; Bagga, P.; Macha, M.A.; Frenneaux, M.P.; Reddy, R.; Haris, M. Non-invasive biomarkers for monitoring the immunotherapeutic response to cancer. J Transl Med. 2020, 18, 471, https://doi.org/10.1186/s12967-020-02656-7.

32. Escanye, J.M.; Canet, D.; Robert, J. Frequency dependence of water proton longitudinal nuclear magnetic relaxation times in mouse tissues at 20 degrees C. Biochim. Biophys. Acta. 1982, 721, 305-11, https://doi.org/10.1016/0167-4889(82)90083-0.

33. Ross, B.; Freeman, D.; Chan, L. Contributions of nuclear magnetic resonance to renal biochemistry. Kidney Int. 1986, 29, 131-41, https://doi.org/10.1038/ki.1986.15.

34. Takeda, M.; Katayama, Y.; Tsutsui, T.; Komeyama, T.; Mizusawa, T.; Saito, K.; Takahashi, H.; Tamaki, M.; Sato, S. Differential diagnosis of solid renal tumors by MRI. Comparison of in situ relaxation times measured with a $0.1 \mathrm{~T}$ imager and histological findings. NMR Biomed. 1993, 6, 329-32, https://doi.org/10.1002/nbm.1940060508.

35. An, Y.; Tang, Q.; Yang, R.; Liu, D.; Zhang, D. In vivo MR imaging of folate-receptor expression with the folate-specific nanospheres in a C6 glioblastoma model. Comput Assist Surg (Abingdon) 2017, 22(supl), 312318, https://doi.org/10.1080/24699322.2017.1389410. 